Supplemental Information

for

\title{
Enhancing the Activity of Insulin at the Receptor Edge: \\ Crystal Structure and Photo-Cross-Linking of A8 Analogues
}

Zhuli Wan ${ }^{1}$, Bin $\mathrm{Xu}^{1}$, Ying-Chi $\mathrm{Chu}^{2}$, Biaoru $\mathrm{Li}^{1}$, Satoe H. Nakagawa ${ }^{3}$, Yan $\mathrm{Qu}^{1}$, Shi-Quan $\mathrm{Hu}^{2}$, Panayotis G. Katsoyannis ${ }^{2}$, and Michael A. Weiss ${ }^{1}$

\section{Table of Contents}

Purpose of Supplement. . . . . . . . . . . . . . . . . . .

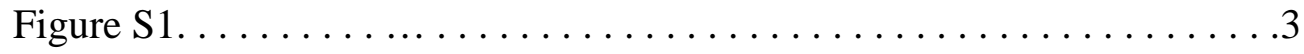

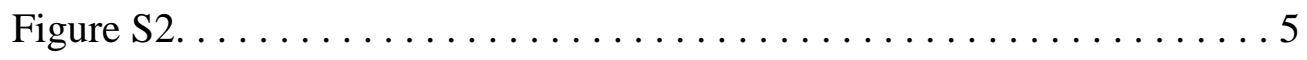

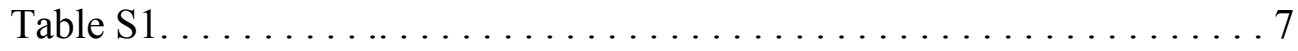

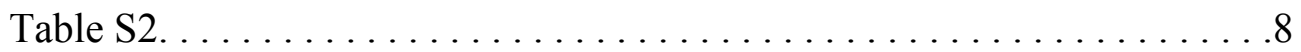

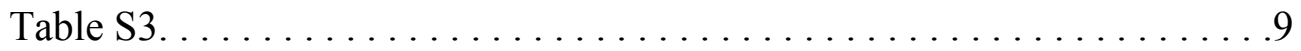

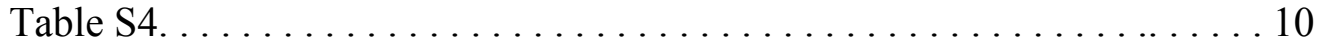

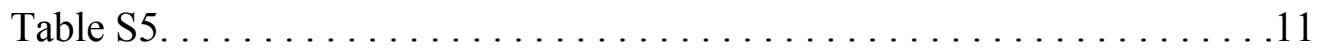




\section{Purpose of Supplement}

The purpose of the Supplement is to provide two figures and five tables. The figures illustrate the Zn-binding sites of the $T_{3} R_{3}{ }^{f}$ coordination geometry model (Fig. S1) and the environment of the phenol molecule bound in the $\mathrm{R}_{3}{ }^{\mathrm{f}}$ trimer (Fig. S2). These features are essentially identical to those observed in nature phenol-stabilized $\mathrm{Zn}$ hexamer. The site of mutation $\left(\mathrm{Thr}^{\mathrm{A} 8} \rightarrow \mathrm{His}\right)$ is on the surface of the hexamer distant from the two $\mathrm{Zn}$-binding sites and three phenol-binding pockets.

Table S1 and S2 provide RMSD values obtained on alignments of the present crystal structure with respect to previous crystal structures of insulin hexamers,. Protomer-specific alignments are provided for the T state (S1) and R state (S2). As controls for the variability observed away native structures, Tables S3 and S4 provide RMSD values on alignment of the present structure with native $\mathrm{T}_{3} \mathrm{R}_{3}^{\mathrm{f}}$ hexamers ( $\mathrm{S} 3$, T-state; and $\mathrm{S} 4$, R-state). Comparison of these RMSD values indicates that $\mathrm{His}^{\mathrm{A} 8}$-insulin falls within the range of native structure, Table S5 provides analogous RMSD values for alignment of the present crystal structure with the solution structure of $[4 \mathrm{E}+\mathrm{A} 8 \mathrm{H}]-$ insulin. 
Figure S1. 2Fo-Fc electron density map contoured at a level of $1.0 \sigma$, showing a phenol molecule bound in the $\mathrm{R}_{3}{ }^{\mathrm{f}}$ trimer of $\mathrm{T}_{3} \mathrm{R}_{3}{ }^{\mathrm{f}} \mathrm{His}^{\mathrm{A} 8}$ - insulin. 


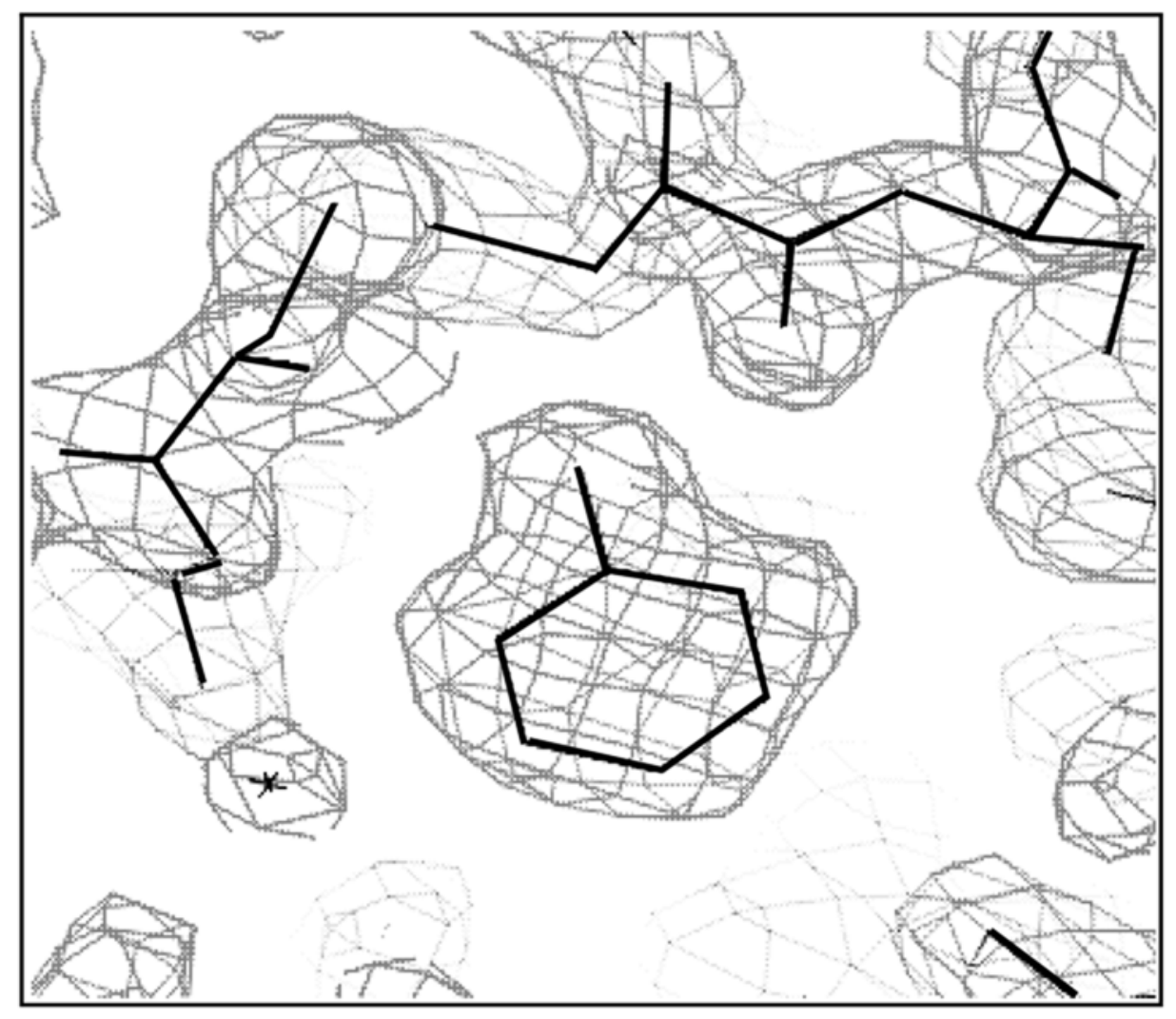

Figure S1 
Figure 2. $2 F o-F c$ electron density map contoured at a level of $1.0 \sigma$, showing the $\mathrm{T} 3$

trimer the coordination geometry is octahedral (A), in the R3f trimer the coordination geometry is tetrahedral (B). 

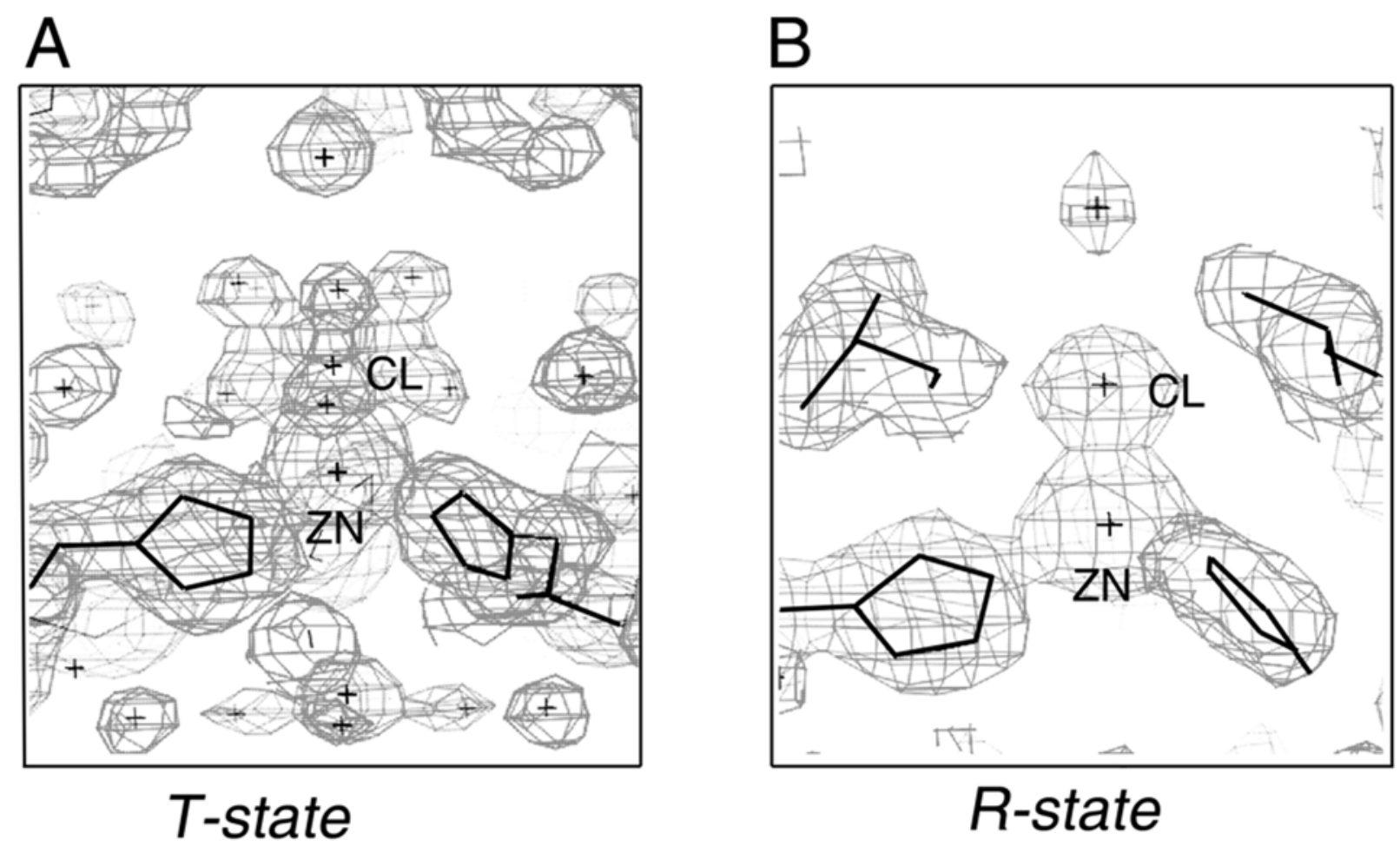

Figure S2 


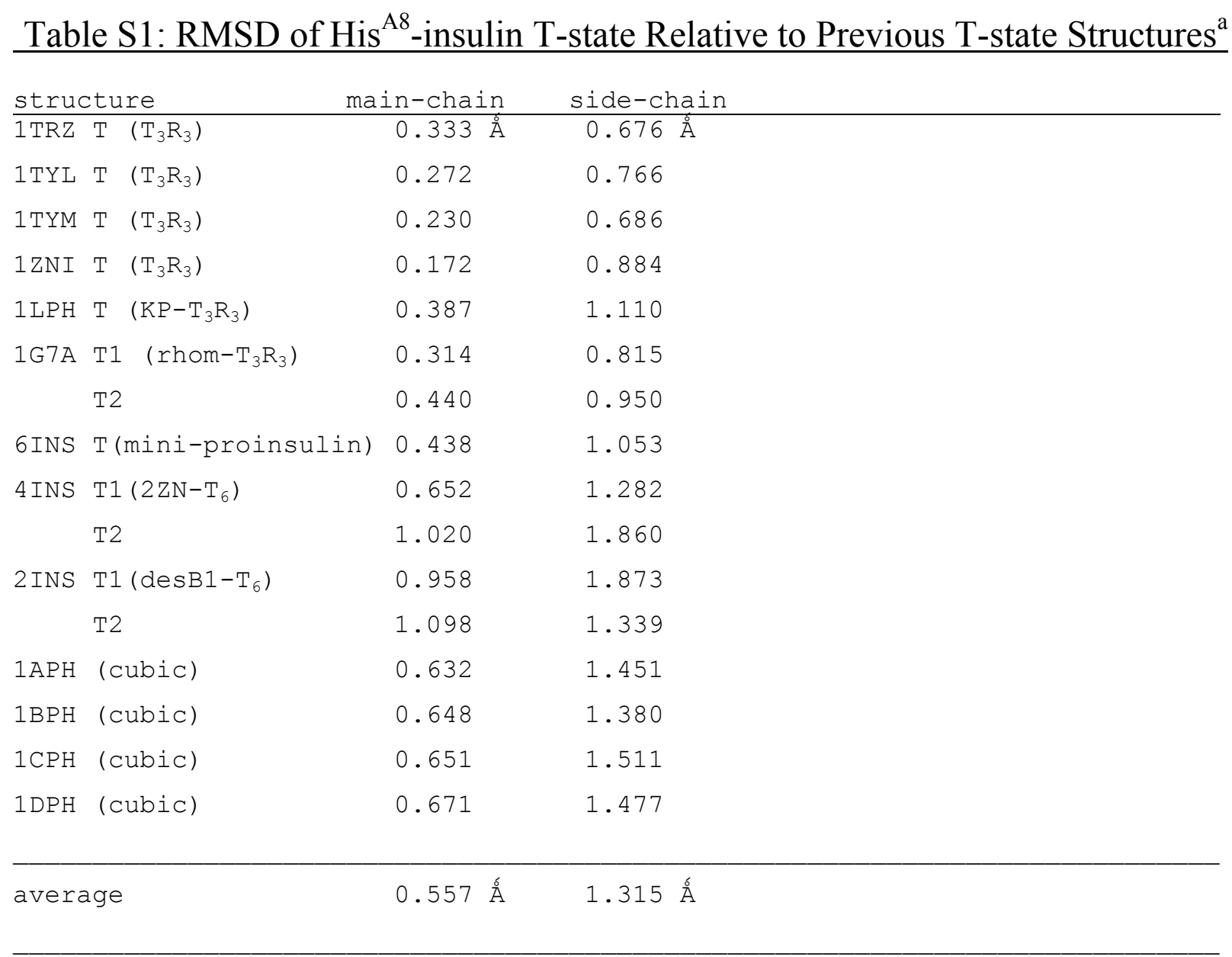

${ }^{\mathrm{a}}$ The structures were aligned according to the main-chain atoms of residue on B3-B28 and A1A21. "KP" designates variant insulin containing substitutions Pro $^{\mathrm{B} 28} \rightarrow$ Lys and Lys ${ }^{\mathrm{B} 29} \rightarrow$ Pro. "rhom" indicates Rhombohedral crystal form."Mini-proinsulin" designate B29-A1 singlechain analog. "desB1" indicates removal of $\mathrm{Phe}^{\mathrm{B} 1}$. 


\section{Table S2. RMSD of His ${ }^{\mathrm{A} 8}$-Insulin R-state Relative to Previous R-state Structures ${ }^{\mathrm{a}}$}

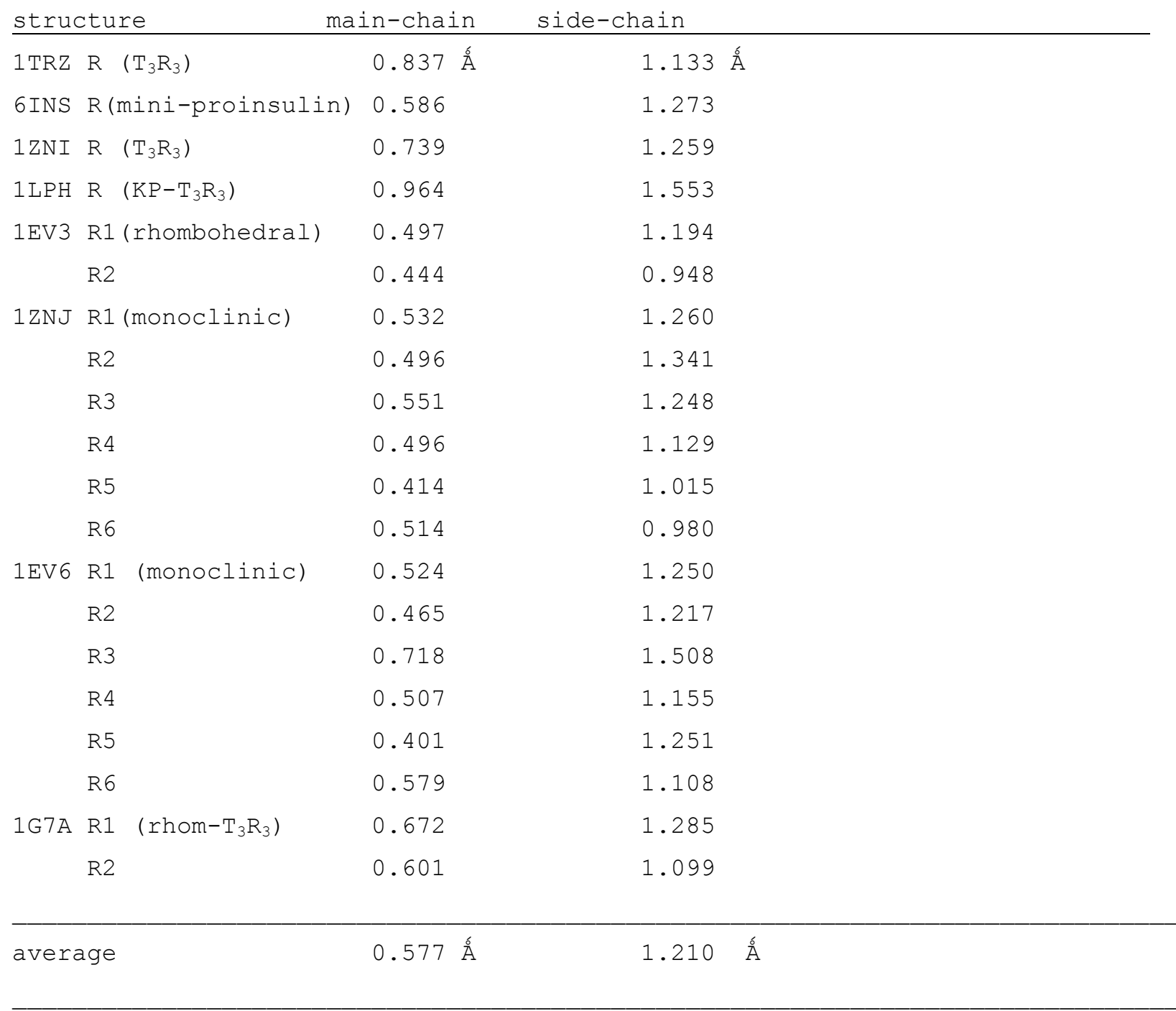

${ }^{\mathrm{a}}$ The structures were aligned according to the main-chain atoms of residue on B3-B28 and A1A21. "KP” designates variant insulin containing substitutions Pro ${ }^{\mathrm{B} 28} \rightarrow$ Lys and Lys ${ }^{\mathrm{B} 29} \rightarrow$ Pro. $^{\circ}$ 
Table S3: RMSD of Native 2-Zinc Insulin T-state with Collection of Previous T-state $\underline{\text { Structures }}^{\mathrm{a}}$

\begin{tabular}{|c|c|c|c|}
\hline \multicolumn{2}{|c|}{ Structure } & \multirow{2}{*}{$\frac{\text { main-chain }}{0.684 \AA ̊}$} & \multirow{2}{*}{$\frac{\text { side-chain }}{1.343 \AA}$} \\
\hline $4 \mathrm{ZN}$ & $\mathrm{T} 2\left(2 \mathrm{ZN}-\mathrm{T}_{6}\right)$ & & \\
\hline \multirow[t]{2}{*}{$2 \mathrm{ZN}$} & $\mathrm{T} 1\left(\operatorname{desB} 1-\mathrm{T}_{6}\right)$ & 0.333 & 0.582 \\
\hline & $\mathrm{T} 2$ & 0.711 & 1.410 \\
\hline $1 \mathrm{APH}$ & $\mathrm{T}$ (cubic) & 0.734 & 1.540 \\
\hline $1 \mathrm{BPH}$ & $\mathrm{T}$ (cubic) & 0.735 & 1.536 \\
\hline $1 \mathrm{CPH}$ & $\mathrm{T}($ cubic) & 0.766 & 1.688 \\
\hline $1 \mathrm{DPH}$ & $\mathrm{T}(\mathrm{cubic})$ & 0.775 & 1.625 \\
\hline $1 \mathrm{TRZ}$ & $\mathrm{T}\left(\mathrm{T}_{3} \mathrm{R}_{3}\right)$ & 0.602 & 1.185 \\
\hline 1TYL & $\mathrm{T}\left(\mathrm{T}_{3} \mathrm{R}_{3}\right)$ & 0.602 & 1.186 \\
\hline $1 \mathrm{TYM}$ & $\mathrm{T}\left(\mathrm{T}_{3} \mathrm{R}_{3}\right)$ & 0.636 & 1.194 \\
\hline $1 \mathrm{ZNI}$ & $\mathrm{T}\left(\mathrm{T}_{3} \mathrm{R}_{3}\right)$ & 0.612 & 1.261 \\
\hline $1 \mathrm{LPH}$ & $\mathrm{T}\left(\mathrm{KP}-\mathrm{T}_{3} \mathrm{R}_{3}\right)$ & 0.627 & 1.253 \\
\hline \multirow[t]{2}{*}{$1 \mathrm{G} 7 \mathrm{~A}$} & $\mathrm{~T} 1\left(\mathrm{rhom}-\mathrm{T}_{3} \mathrm{R}_{3}\right)$ & 0.687 & 1.355 \\
\hline & $\mathrm{T} 2$ & 0.714 & 1.384 \\
\hline \multicolumn{2}{|c|}{ average } & $0.658 \AA$ & $1.324 \AA$ \\
\hline
\end{tabular}

\footnotetext{
a The structures were aligned according to the main-chain atoms of residue on B3-B28 and A1-A21. “KP” designates variant insulin containing substitutions Pro $^{\mathrm{B} 28} \rightarrow$ Lys and Lys ${ }^{\mathrm{B} 29} \rightarrow$ Pro. "rhom" indicates Rhombohedral crystal form. “desB1" indicates removal of Phe ${ }^{\mathrm{B} 1}$.
} 


\section{Table S4. RMSD of Native Insulin R-state (1TRZ) Relative to Collection of Previous}

\section{$\underline{\mathrm{R}_{\text {-state Structures }}^{\mathrm{a}}}$}

\begin{tabular}{|c|c|c|c|}
\hline & structure & main-chain & side-chain \\
\hline \multirow[t]{6}{*}{$1 \mathrm{EV} 6$} & R1 (monoclinic) & $0.851 \AA ̊$ & $1.350 \AA ̊$ \\
\hline & $\mathrm{R} 2$ & 0.731 & 1.167 \\
\hline & R3 & 0.994 & 1.567 \\
\hline & $\mathrm{R} 4$ & 0.759 & 1.199 \\
\hline & $\mathrm{R} 5$ & 0.748 & 1.410 \\
\hline & R6 & 0.728 & 1.243 \\
\hline \multirow[t]{6}{*}{$1 \mathrm{ZNJ}$} & R1 (monoclinic) & 0.779 & 1.225 \\
\hline & $\mathrm{R} 2$ & 0.694 & 1.422 \\
\hline & $\mathrm{R} 3$ & 0.783 & 1.389 \\
\hline & $\mathrm{R} 4$ & 0.713 & 1.032 \\
\hline & R5 & 0.760 & 1.228 \\
\hline & R6 & 0.747 & 1.193 \\
\hline \multirow[t]{2}{*}{$1 \mathrm{G} 7 \mathrm{~A}$} & $\mathrm{R} 1\left(\right.$ rhom $-\mathrm{T}_{3} \mathrm{R}_{3}$ ) & 0.535 & 1.031 \\
\hline & $\mathrm{R} 2$ & 0.779 & 1.246 \\
\hline $1 \mathrm{LPH}$ & $\mathrm{R}\left(\mathrm{KP}-\mathrm{T}_{3} \mathrm{R}_{3}\right)$ & 0.535 & 0.997 \\
\hline $1 \mathrm{ZNI}$ & $\mathrm{R}\left(\mathrm{T}_{3} \mathrm{R}_{3}\right)$ & 0.209 & 0.573 \\
\hline average & & $0.622 \AA ̊$ & $1.205 \AA ̊$ \\
\hline
\end{tabular}

${ }^{\mathrm{a}}$ The structures were aligned according to the main-chain atoms of residue on B3-B28 and A1-A21. “KP” designates variant insulin containing substitutions Pro $^{\mathrm{B} 28} \rightarrow$ Lys and Lys ${ }^{\mathrm{B} 29} \rightarrow$ Pro. 


\begin{tabular}{|c|c|c|}
\hline structure & main-chain & side-chain \\
\hline NMR model-1 & $1.547 \AA$ & $2.438 \AA$ \\
\hline NMR model-2 & 2.060 & 2.870 \\
\hline NMR model-3 & 1.688 & 2.619 \\
\hline NMR model-4 & 1.952 & 2.681 \\
\hline NMR model-5 & 1.794 & 2.698 \\
\hline NMR model-6 & 2.067 & 2.864 \\
\hline NMR model-7 & 1.818 & 2.907 \\
\hline NMR model-8 & 1.638 & 2.607 \\
\hline NMR model-9 & 1.770 & 2.703 \\
\hline NMR model-10 & 1.511 & 2.358 \\
\hline NMR model-11 & 1.901 & 2.925 \\
\hline NMR model-12 & 2.027 & 2.788 \\
\hline NMR model-13 & 1.739 & 2.871 \\
\hline NMR model-14 & 1.815 & 2.722 \\
\hline NMR model-15 & 1.862 & 2.785 \\
\hline NMR model-16 & 1.648 & 2.475 \\
\hline NMR model-17 & 2.272 & 3.099 \\
\hline NMR model-18 & 1.779 & 2.833 \\
\hline NMR model-19 & 1.979 & 2.803 \\
\hline NMR model-20 & 1.865 & 2.724 \\
\hline NMR model-21 & 1.634 & 2.652 \\
\hline NMR model-22 & 1.679 & 2.518 \\
\hline NMR model-23 & 1.639 & 2.561 \\
\hline NMR model-24 & 1.499 & 2.599 \\
\hline NMR model-25 & 1.826 & 2.662 \\
\hline NMR model-26 & 2.097 & 2.490 \\
\hline average & $1.812 \AA$ & $2.702 \AA$ \\
\hline
\end{tabular}

${ }^{\mathrm{a}}$ The structures were aligned according to the main-chain atoms of residue on B3-B28 and A1-A21. 